\title{
Dynamic Channel Distribution with Power Sharing and Bit Insertion for Downlink Multiuser OFDMA
}

\author{
Kimmi Salaria \\ Department of E.C.E \\ Lovely Professional UniversityPhagwara, India
}

\begin{abstract}
An imminent mobile and telecommunication system must sustain not only accepted speech services however several other benefits such as wireless multimedia, small circuit switching and packet dependent data transmission are also being considered. For the realization of the above services with an attractive modulation procedure whose properties makes it possible known as Orthogonal frequency division multiplexing access (OFDMA). And to enhance the system performance a power sharing and bit insertion technique is being investigated. There are various NP-complete and IP problems but the most bit loading in addition with subcarrier allocation problems were being formulated. Also, one more technique which was based on variable amplitude of subcarriers (VAS). We can curb the global power transmission underneath the data rate constraint and exploit the data rate constraint beneath the power constraint. With this users can be allotted to high channel gain and the result of this was efficiently improvement in spectrum utilization. Algorithm which distinctively perform power and rate optimization under some constraint known as margin adaptive and rate adaptive optimization problems respectively in Multiuser for BER $=10^{-4}$ and number of subcarrier $: \geq 64$.
\end{abstract} Keywords

Multiuser OFDM, Subcarrier allocation, Margin Adaptive optimization, Rate adaptive optimization, power sharing.

\section{INTRODUCTION}

In the last decade, communication system undergoes remarkable growth globally. Forthcoming wireless technology are anticipated to equipped with a ample variety of new services ranging from voice to high quality wireless multimedia at the least possible cost [1]. The magnificent user interest in multimedia is powering the need for data rates which was quite high than previous available data rates. To support applications having high data rate under restricted radio resources and rough wireless channel conditions mainly dynamic channel allocation having capability of both higher spectral efficiency as well as better QOS [1].

With OFDM technique, a set of orthogonal subcarriers having narrowband was inherited from wireless broadband channel [8]. In view of fact that the channel frequency response in single user systems are different at various subcarriers and to increase the system performance at this stage adapting the modulation in relation with power level over each subcarrier [2]. Also diverse subcarriers in an OFDM may contain dissimilar fading gains in an available channel access, utilization of same modulation in every subcarrier may leads to ineffective use of overall spectrum [2].

Since multiuser OFDM are designed with bit insertion and subcarrier allocation schemes in which transmit power seems to be minimized under data rate constraint and also data rate are enhanced to be maximized for the power constraint [3]. The non-linear techniques are being transformed for linear optimization using IP [3]. OFDM has the capacity to tackle

\author{
B. Arun Kumar \\ Department of E.C.E \\ Lovely Professional University \\ Phagwara, India
}

intersymbol interference above multipath fading channels. OFDM depended manifold access technique capably utilizes restricted bandwidth and transfer power in broad band transmission. In multiuser OFDM, complete spectrum is assigned to many users on the basis of some algorithm. On the contrary each user is assigned with single subcarrier and they are incident to distinct number of fades and transfer different bits. Instead of this if the subcarrier is tend to appear in one fade this is not the reason to be engaged for the other user. An important factor for the fading is distance from the BS and the broadcast power level is arranged in view of the distance from base station. One proposed solution for this problem is that if we get the channel state information (CSI) than we can assign subcarriers to users and this allocation scheme is known as adaptive multiple user variable amplitude subcarriers (VAS) [4].

In precise, transmitter can transmit higher transmission rates modulated on subcarriers with healthier channel conditions instead lesser rates or no information bits on subcarriers in deep fade [2]. If we consider that CSI for every subcarrier is available on the transmitter in addition with different power, bit and schemes for both power and bit insertion has been proposed in the attainment of different channel improvement parameters in respect to different constraint conditions. This work has been continued till the minimizing of power as well as BER is achieved. As water filling theorem to maximize spectral efficiency in terms of data rate and adapted transmit power [2].

In this paper, Section 2 will focus upon the System Parameters for rate, SNR and bit loading in OFDMA, Section 3 will light upon, Section 4 will give us about the plot of various system parameters and Section 5 will conclude the paper.

\section{SYSTEM PARAMETERS IN OFDMA}

The parameters which solely describes the subcarrier allocation in multiuser OFDMA can be represented as follows [1][2][5][6] :

$h_{k}: \rightarrow$ channel attenuation

$n_{k}: \rightarrow$ additive white Gaussian noise (AWGN)

$T_{\text {load }}: \rightarrow$ traffic load determines relative amount of resources which are at present in service.

$G \quad: \rightarrow$ average reuse existing in the system.

$K \quad: \rightarrow$ total number of users.

$R_{k} \quad \rightarrow$ data rate of the $\mathrm{k}^{\text {th }}$ user.

$B: \rightarrow$ bandwidth in MHz.

$N_{\text {tot }}: \rightarrow$ total amount of available subchannel.

$S \quad: \rightarrow$ spectral efficiency. 
$C_{k, n}: \rightarrow$ number of information bits of a $\mathrm{k}^{\text {th }}$ user which are transfer to $\mathrm{n}^{\text {th }}$ subcarrier.

$\alpha_{k, n}: \rightarrow$ channel gain constraint for user $\mathrm{k}$ possess subchannel $\mathrm{n}$.

$P_{k, n}: \rightarrow$ power assigned to user $\mathrm{k}$ having subcarrier $\mathrm{n}$.

$P_{T}: \rightarrow$ total transmission power of system.

$\Gamma_{k, n}: \rightarrow$ SNR for $\mathrm{k}^{\text {th }}$ user partake of subcarrier $\mathrm{n}$.

\section{DYNAMIC SHARING METHODS}

\subsection{Margin Adaptive}

It tends to shrink the complete transmission power needed for transmitting the information with rate $\left\{R_{1} \ldots \ldots . R_{k}\right\}$ will be given as [3]:

$$
\min _{C_{k, n}, P_{k, n}} P_{T}=\min _{C_{k, n}, P_{k, n}} \sum_{k=1}^{K} \sum_{n=1}^{N} \frac{f_{k}\left(C_{k, n}\right)}{\alpha_{k, n}} \cdot \rho_{k, n}
$$

Subject to

$$
R_{k}=\sum_{n=1}^{N} C_{k, n} \rho_{k, n}
$$

\subsection{Rate Adaptive}

It has a bias to maximizes the throughput of least amount of users $\mathrm{k}$ and subject to the condition of power constraint [1][3]

$$
\max _{C_{k, n}, P_{k, n}} \min _{k} R_{k}=\max _{C_{k, n}, P_{k, n}} \min _{k} \sum_{n=1}^{N} C_{k, n} \cdot \rho_{k, n}
$$

Subject to

$$
\sum_{k=1}^{K} \sum_{n=1}^{N} \frac{f_{k}\left(C_{k, n}\right)}{\alpha_{k, n}^{2}} . \rho_{k, n} \leq P_{T}
$$

These techniques have a propensity to allocate more transmission power to all users having bad channel response.

\subsection{Resource Allocation}

As BER is considered to be a function of SNR which is equal to $E_{b} / N_{o}$, where $E_{b}$ is defined to be signal power and $N_{o}$ is a noise power density [7]. Also take into account modulation schemes for QPSK and QAM as mentioned below [7].

$$
\begin{aligned}
& P_{e}(Q P S K)=Q\left[\left(2 E_{b} / N_{o}\right)^{1 / 2}\right] \\
& P_{e}(16-Q A M)=(3 / 4) Q\left[\left(4 E_{b} / 5 N_{o}\right)^{1 / 2}\right] \\
& P_{e}(64-Q A M)=(7 / 12) Q\left[\left(2 E_{b} / 7 N_{o}\right)^{1 / 2}\right]
\end{aligned}
$$

Steps of resource allocation Algorithm:

1. Start for $\mathrm{v}=1$ to $\mathrm{V}$

2. Find optimal modulation using eq.(3),(4),(5)

$$
M_{\text {opt }}(v) \text {. }
$$

3. Determine optimal amount of information bits to modulate on each subcarrier.

$$
m_{\text {opt }}(v)=\log _{2}\left(M_{\text {opt }}(v)\right)
$$

4. Figure out primary amount of available channels using

$$
S_{k}=\frac{R_{k}}{m_{\text {opt }}(k)}
$$

5. The whole number of subcarriers must not go beyond the accessible number of subcarrier $\mathrm{N}$

$$
\text { while }\left(\sum_{v=1}^{V} S_{v} \geq N\right)
$$

6. Finally the users with least amount of allocated subcarrier are made to be blocked and get

$$
\begin{gathered}
v_{o}=\arg \min S_{v} \\
S_{v_{o}}=0
\end{gathered}
$$

7. End of algorithm.

\subsection{Subcarrier Assignment}

In this method, certain number of subcarriers is allocated to specific station, in cooperation with some quantity of bits and power on every subcarrier. For this we consider an assignment vector $A=\left[A_{n}\right]$ where each $A_{n}$ consists of user and they are allotted channel $\mathrm{n}$ and a gain matrix $G=\left[G_{v, n}\right][7]$

Steps for subcarrier assignment algorithm [7]:

1. Start for $\mathrm{n}=1$ to $\mathrm{N}$.

2. Determine

$$
v_{o}=\arg \max G_{v, n}
$$

3. While

$$
S_{v_{o}}=0 \& G_{v_{o}, n} \neq 0
$$

4. Assign $G_{v_{o}, n}=0$

5. Compute the factor

$$
v_{o}=\arg \max G_{v, n}
$$

6. Now assign the channel $\mathrm{n}$ to the user $v_{o}$

$$
\text { and } S_{v_{o}}=S_{v_{o}}-1
$$

7. End of algorithm

\subsection{Variable Amplitude of Subcarriers (VAS)}

The subcarriers can be allocated to number of users on the basis of immediate channel state information (CSI) and power can be raised for the customers who are passing the reference boundary(RB) as well as meeting end of the cell known as footprint [4].

Primary signal of $\mathrm{n}^{\text {th }}$ subcarrier [4]:

$$
S_{n}(t)=X_{n}(t)[\cos (2 \pi f t) \pm j \sin (2 \pi f t)]
$$

Now, compute transformation by using N-pt. IFFT moreover the signal is characterize as [4]: 


$$
S(t)=\frac{1}{N} \sum_{n=0}^{N} X_{n}(t)[\cos (2 \pi f t) \pm j \sin (2 \pi f t)]
$$

Also, signal to noise ratio (SNR) for $\mathrm{k}^{\text {th }}$ user having $\mathrm{n}^{\text {th }}$ subcarrier can be formulated as [6]:

$$
S N R_{k} \operatorname{or}_{k}=\frac{P_{r}(k, n, d)}{I+\left(\frac{B^{*} N_{o}}{N}\right)}
$$

Finally, to approximate the system channel gain in terms of distance between the user $\mathrm{k}$ and base station (BS) is given as [4]:

$$
P_{r}(k, n, d)=d^{-v} * P_{t}
$$

1. Compute all users $\mathrm{k}$ which are moving closer to footprint (FP) and are trying to cross reference boundary (RB).

2. Determine those users $\mathrm{k}$ which are on the way to BS.

3. Evaluate B.E.R for all $\mathrm{k}$ which are completely depending on SNR.

$$
B E R=0.5 * \operatorname{erfc}(\sqrt{S N R})
$$

4. Now in view of immediate CSI, find SNR levels of every user which are straight forwardly depends upon distance of customers form the BS.

5. VAS are being allocated to the users with respect to the distance from BS.

Allocation scheme for VAS [4]:

\section{DYNAMIC BIT INSERTION}

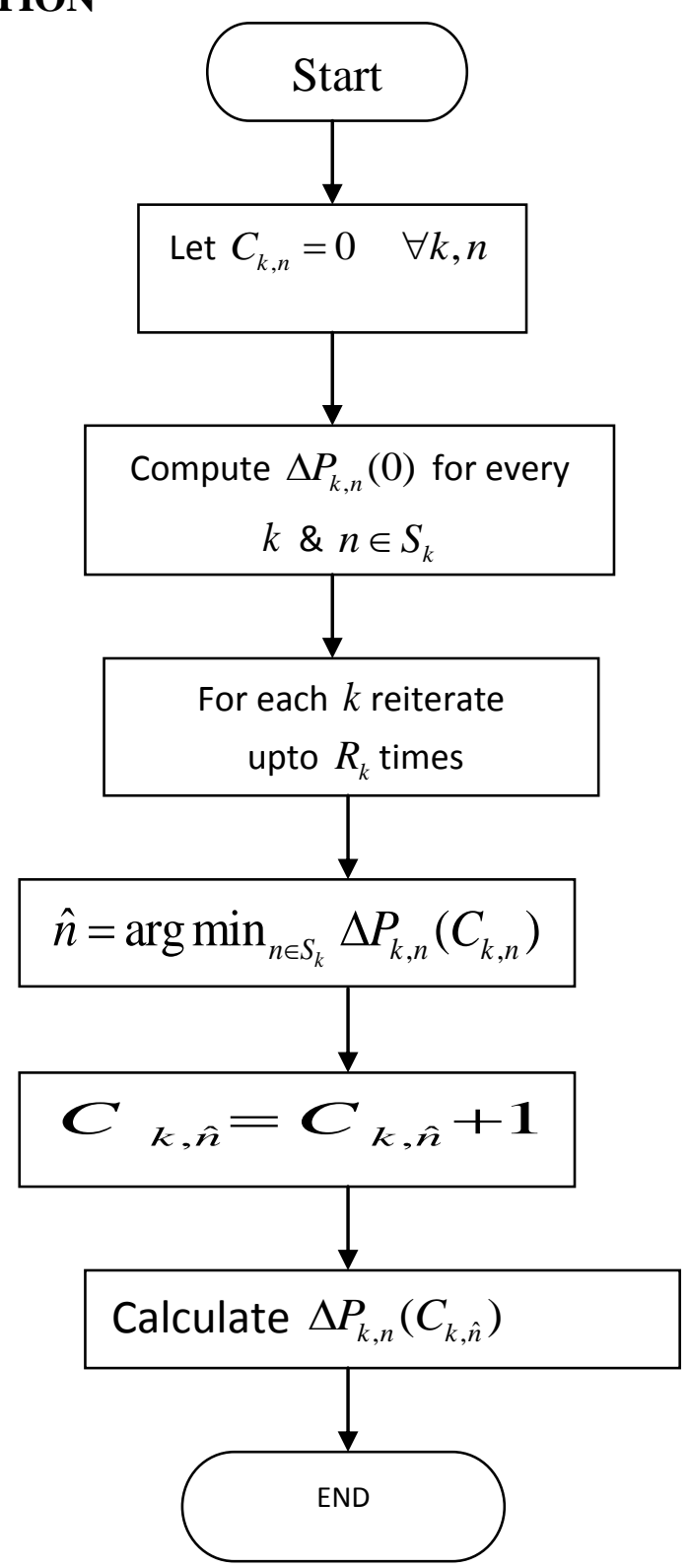

Fig 1: Flow chart for Bit insertion scheme 
As bit insertion is done under the notion that channel assignment to various user has been completed and for this greedy algorithm has been used which states that allocate bits to subcarrier of user one at a time and in every allocation we select those subcarrier which need smallest amount of additional power [3] and this power can be represented as :

$$
\Delta P_{k, n}(c)=\left[f_{k}(c+1)-f_{k}(c)\right] / \alpha_{k, n}^{2}
$$

\section{SIMULATION RESULTS}

We compare the BER and SNR of the multiuser OFDMA and it is $10^{-4}$ and in Fig. 2 it is corresponds to SNR for $\mathrm{N}=64$. After while we plot the graph for SNR vs Bandwidth of carrier frequency offset and this can result in the number of users for getting subcarriers in Fig. 3.

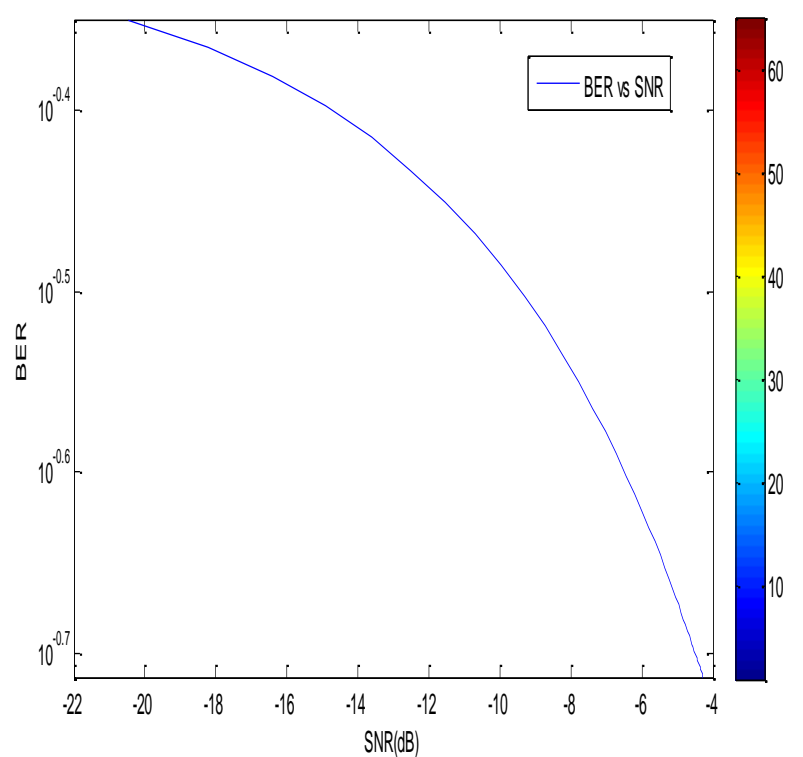

Fig 2: Graph between BER and SNR

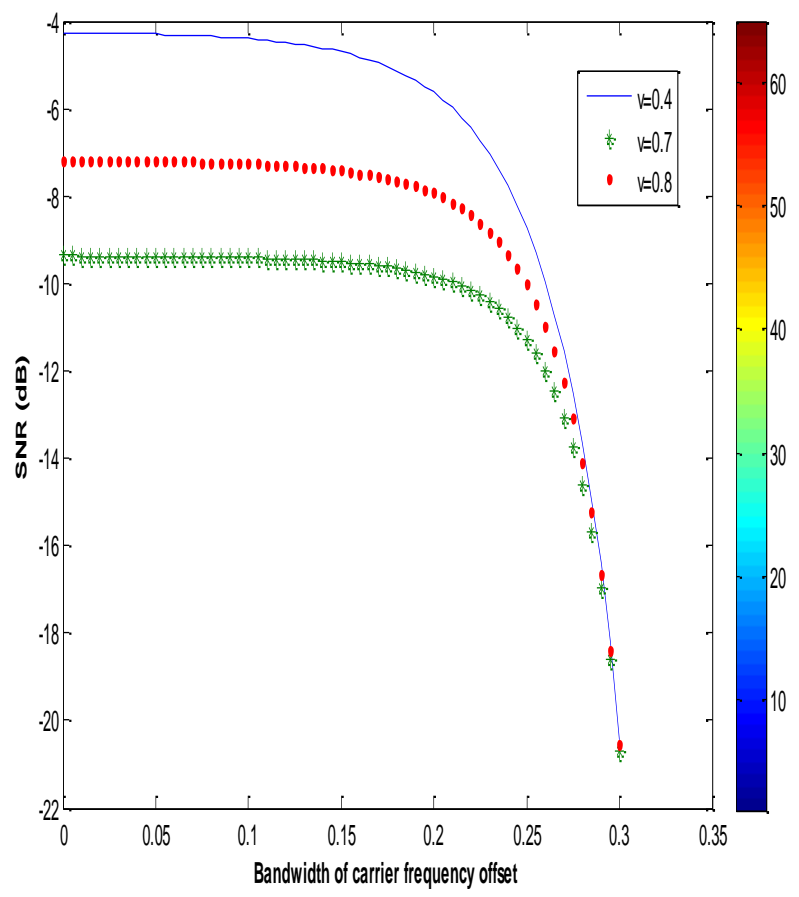

Fig 3: Graph in between SNR and BW of carrier frequency offset

\section{CONCLUSION}

We have extend the various dynamic power sharing schemes and implemented the effect of SNR over BER through MATLAB. Instead we also find how the bandwidth of carrier frequency offset effect the SNR in multiuser OFDMA as such as shown in Fig.2 and Fig.3. It can observe that the $\mathrm{x}$-axis depicts the value of 'SNR' and y-axis shows the BER being engaged from the transmitter to the receiver through channel. Also, the blue line correspond to $\mathrm{v}=0.4$, the green star line corresponds $\mathrm{v}=0.7$ and the red star line corresponds to $\mathrm{v}=0.8$ moreover all these signify different variance of phase noise of the channel which would necessary for channel allocation and bit insertion. All the above dynamic sharing methods for channel allocation and dynamic bit loading scheme as shown in flowchart will certainly work as an OFDM dynamic resource allocation.

\section{ACKNOWLEDGEMENTS}

Our valuable thankfulness wished for mentor and related persons because without their support, it is hard to complete the work represented in this paper.

\section{REFERENCES}

[1] Khaled Ben Letaif, Ying Jun(Angela) Zhang, "DYNAMIC MULTIUSER RESOURCE ALLOCATION AND ADAPTION FOR WIRELESS SYSTEMS". IEEE Wireless Communications, pp. 38-47, August 2006.

[2] Liexin Peng, Jian Du and Guangxi Zhu, "Adaptive Resource allocation and Scheduling for the Delay Limited OFDM Systems". IEEE Conference on Local Computer Networks, pp.731-736, 2007.

[3] Inhyoung Kim, Hae Leem Lee, Beomsup Kim and Yong H.Lee, "On the Use of Linear Programming for Dynamic Subchannel and Bit Allocation in Multiuser OFDM". IEEE , pp.3648-3652, 2001.

[4] Vidya Kant Dwivedi, Shivesh Tripathi, Vijay Shanker Tripathi, Rajeev Tripathi and Sudarshan Tiwari, "Shared Power Allocation among Subcarriers of OFDM Systems". International Conference on Emerging Trends in Electronic and Photonic Devices and Systems, pp.266269, 2009.

[5] M. Wahlqvist, H. Olofsson, M. Ericson, C. Ostberg and R.Larsson, "CAPACITY COMPARISON OF AN OFDM BASED MULTIPLE ACCESS USING DIFFERENT DYNAMIC RESOURCE ALLOCATION”. IEEE, pp.1664-1668, 1997.

[6] Jia Tang and Xi Zhang, "Cross - Layer Design of Dynamic Resource Allocation with Diverse QOS Guarantees for MIMO-OFDM Wireless Networks". IEEE International Symposium on a World of Wireless Mobile and Multimedia Networks, 2005.

[7] Adil EL Bourichi and Hiroto Yasuura, "A Low Complexity and Energy Efficient Dynamic Channel Allocation Algorithm for Multiuser OFDM". IEEE, 2007.

[8] Jurong Bai, Yafang Yin and Yanben Wang, "Simulation of Dynamic Resource allocation in OFDMA Systems". Cross Strait Quad-Regional Radio Science and Wireless Technology Conference, pp.839-842, July 26, 2011. 